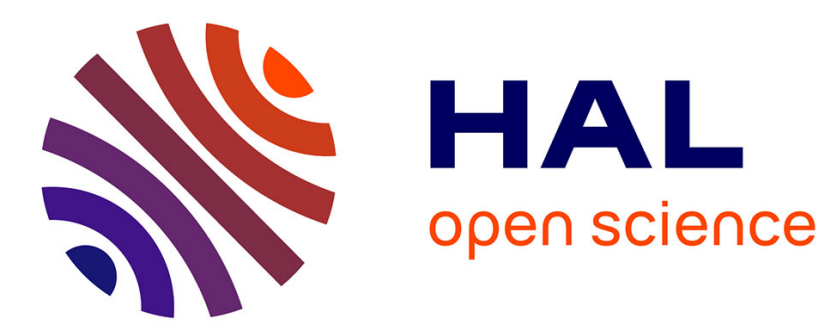

\title{
Effective medium-based optical real-time control over plasmon tuning in laser-induced nanocomposites
}

\author{
Tatiana E Itina, Maxim Sergeev, Roman Zakoldaev
}

\section{To cite this version:}

Tatiana E Itina, Maxim Sergeev, Roman Zakoldaev. Effective medium-based optical real-time control over plasmon tuning in laser-induced nanocomposites. SPIE LASE, Mar 2021, Online Only, France. $10.1117 / 12.2583243$. hal-03413598

\section{HAL Id: hal-03413598 \\ https://hal.science/hal-03413598}

Submitted on 3 Nov 2021

HAL is a multi-disciplinary open access archive for the deposit and dissemination of scientific research documents, whether they are published or not. The documents may come from teaching and research institutions in France or abroad, or from public or private research centers.
L'archive ouverte pluridisciplinaire HAL, est destinée au dépôt et à la diffusion de documents scientifiques de niveau recherche, publiés ou non, émanant des établissements d'enseignement et de recherche français ou étrangers, des laboratoires publics ou privés. 


\title{
Effective medium-based optical real-time control over plasmon tuning in laser-induced nanocomposites
}

\author{
Tatiana E. Itina*a,b, Maksim Sergeev ${ }^{\mathrm{b}}$, Roman Zakoldaev ${ }^{\mathrm{b}}$ \\ ${ }^{a}$ Laboratoire Hubert Curien, UMR CNRS 5516, Université Jean Monnet, Universite de Lyon, Saint- \\ Etienne, France; ${ }^{\text {b }}$ TTMO University, Kronverkskiy Pr. 49, Saint Petersburg, Russia
}

\begin{abstract}
Laser-induced structuring of nanoporous glass composites is promising for numerous emerging applications in photonics, plasmonics, and medicine. In these laser interactions, an interplay of photo-thermo-chemical mechanisms is commonly activated and is extremely difficult to control. The choice of optimum laser parameters to tune the resulted optical properties remains extremely challenging. In this paper, we analyze the mechanisms involved and propose a way to control not only structures formed by laser inside nanoporous glass composites doped by metallic ions and nanoparticles, but also their plasmonic properties. For this, both experimental and numerical approaches are combined. The transmitted and reflected laser powers are used to analyze the modification process. Spectral microanalysis provides plasmonic properties. Numerical effective medium modeling connects the measured data to the estimated size, concentration, and chemical composition of the second phase across the initial sample.
\end{abstract}

Keywords: Laser, nanoparticles, nanostructures, modeling, plasmonic properties, control, monitoring, nanocomposite

\section{INTRODUCTION}

Nanocomposite materials based on dielectric and semiconductor matrices, e.g. $\mathrm{SiO}_{2}$ and $\mathrm{TiO}_{2}$, with plasmonic nanoparticles have found numerous applications in optics, sensing, photocatalysis, in the development of solar cells, and image recording for security ${ }^{1}$. Laser is an efficient tool allowing promising possibilities of the synthesis of such materials. However, both the fabrication of optical nanocomposites and an efficient control over their optical properties remain challenging.

Optical properties of nanocomposite materials rely on the plasmonic properties of metallic nanoparticles, which depend on the shape, size, and dispersion of nanoparticles, as well as on the optical properties of the surrounding media ${ }^{2,3}$. Herein, effective medium approach in various approximations ${ }^{4,5}$ is used to simulate and predict the main optical characteristics of such materials. In the approximations, the volume fraction of the metal phase is used, however, the experimental determination of this parameter remains difficult, and the calculation methods are not fully described in the literature.

In this paper, we couple the effective medium theory in the Bruggeman-Bergman approximation for the calculations of the spectral characteristics of composite materials based on solid-state thin film with spherical silver nanoparticles. The volume fraction of the metal phase for determining the spectral characteristics are calculated taking into account the size dispersion of nanoparticles. We considered all the metallic inclusions to be conventionally divided into three ensembles: nanoclusters (atomic radius smaller than $1 \mathrm{~nm}$ ), seeds (from 1 to $3 \mathrm{~nm}$ ), and nanoparticles (from 3 to $50 \mathrm{~nm}$ ). The effect of nanoparticles dispersion and the radius on volume fraction of the metal phase and spectral characteristics are demonstrated. The simulation results are compared with experimental data of nanoparticles size distribution in $\mathrm{TiO}_{2}$ and $\mathrm{SiO}_{2}$ films containing silver nanoparticles and irradiated by UV laser. The obtained results, if combined with the measurements of the reflected and transmitted laser power, are suitable for the real-time control over the fabricated nanocompostes. 


\subsection{Laser-assisted nanocomposite synthesis and characterization}

For the experiment, mesoporous thin films are firstly preparation by using either sol-gel or a complex and multistage procedure, and the obtained films are impregnated with silver ions by soaking in salts as described elsewere ${ }^{6,7,8}$. As a result, $\mathrm{SiO} 2$ and $\mathrm{TiO} 2$ matrices with embedded small (around $3 \mathrm{~nm}$ ) Ag nanoclusters were obtained. Several additional samples, such as porous glasses without dopants, fully sintered glasses, and fused silica, are applied as the reference samples for the simulations. Sample transmittance and reflectance are measured in the range from 0.3 to $1.1 \mu \mathrm{m}$ by a spectrophotometer (MSFU-K Yu-30.54.072, LOMO, St. Petersburg, Russia), where the minimum registration region is equal to $2 \mu \mathrm{m}$. The transmittance $T_{\text {meas }}$ and reflectance $R_{\text {meas }}$ of all the samples are measured at normal incidence of light. Spectral curves for the initial porous glass are similar to these of fused silica, except for the UV absorption range. Introducing halides in the porous results in a significant absorption in the range from 0.45 to $0.7 \mu \mathrm{m}$. The preliminary spectral samples characterization helps us to choose the correct laser source wavelength for processing inside the sample.

In one part of the experiments, $\mathrm{CW}$ fiber laser source is chosen to induce both silica matrix and salt mixture modification triggering local optical changes inside GC. The wavelength $(\lambda=1064 \mathrm{~nm})$ differs from the maximum absorption of samples and enables gentle processing. In the other part, shorter wavelength, $\lambda=405 \mathrm{~nm}$, is applied and is in resonance with silver plasmonic nanoparticle absorption. $\mathrm{TiO}_{2}$ and $\mathrm{SiO}_{2}$-based nanocomposite films with silver nanoparticles were synthesized. The transmittance $T_{\text {meas }}$ and reflectance $R_{\text {meas }}$ of all the samples are registered during laser irradiation. SEM microscopy is used to analyze the obtained modifications, nanoparticle mean size and size distributions.

\subsection{Effective-medium- based model}

The spectral parameters of the nanocomposite samples can be calculated based on the dielectric function. In the case of a composite material with spherical nanoparticles, the model of effective media in the Bruggeman-Bergman approximation was used as follows

$$
\begin{gathered}
\varepsilon_{e f f}(\lambda)=\varepsilon_{\text {mat }}(\lambda) \cdot\left[1-v_{n p} \int_{0}^{1} \frac{g\left(u, v_{n p}\right)}{\varepsilon_{\text {mat }}(\lambda) /\left[\varepsilon_{\text {mat }}(\lambda)-\varepsilon_{\text {me }}(\lambda)\right]-u} d u\right], \\
\varepsilon_{m e}(\lambda)=\varepsilon_{b}(\lambda)+\frac{\omega_{p}^{2}}{\omega}\left[\frac{1}{\omega+i \Gamma_{b}}-\frac{1}{\omega+i \Gamma}\right], \\
\Gamma=\Gamma_{b}+\frac{4}{3} \frac{v_{F}}{R},
\end{gathered}
$$

where $g\left(u, v_{n p}\right)$ is spectral density function, $u R / L\left(v_{n p}\right)$ is right and left parts of the volume fraction, $\varepsilon_{m a t}(\lambda)$ is the permittivity of the surrounding medium, $\varepsilon_{\text {mat }}(\lambda)$ is the permittivity of the nanoparticles, $\varepsilon_{b}(\lambda)$ is the permittivity of the bulk metal, $\hbar \omega_{p}=9.2 \mathrm{eV}$ is the plasma frequency, $\Gamma_{b}=3.464 \cdot 1013 \mathrm{~s}^{-1}$ is the electron scattering rate. $\Gamma$ is a coefficient, $v_{F}$ is a Fermi velocity, and $R$ is an average radius of spherical nanoparticles. To determine the volume fraction of the metal phase, $v_{n p}$, the initial distribution of all metallic inclusions was separated into three groups: nanoclusters ( $r a t$ is smaller than $1 \mathrm{~nm}$ ), nucleuses (from 1 to $3 \mathrm{~nm}$ ), and nanoparticles (from 3 to $50 \mathrm{~nm}$ ).

We considered only nanoparticles to influence the spectral characteristics of the nanocomposite. Thus, the volume fraction was calculated by using the following equations

$$
v_{n p}=\frac{4 \pi}{3} r_{a t}^{3} \int_{r_{n p}} C_{a}(r) d r,
$$




$$
\begin{gathered}
C_{a}(r)=K_{c} C_{\text {Atom }}\left(\frac{r}{r_{a t}}\right)^{3} C_{p}(r), \\
\frac{1}{K_{c}}=\int_{r_{n c}}\left(\frac{r}{r_{a t}}\right)^{3} C_{p}(r) d r+\int_{r_{m a c}}\left(\frac{r}{r_{a t}}\right)^{3} C_{p}(r) d r+\int_{r_{n p}}\left(\frac{r}{r_{a t}}\right)^{3} C_{p}(r) d r .
\end{gathered}
$$

where $r_{a t}$ is an atomic radius of metal, $C_{a}$ is the atoms size distribution, $C_{p}$ is the initial nanoparticles size distribution, and $K_{c}$ is the normalizing constant. Equations (4) - (6) show that the initial size distribution of nanoparticles, $C_{p}$, affects the volume fraction of the metal phase. Diffusional growth of nanoparticles was simulated by using the following equation

$$
r_{a v t}(T, t)=\left[r_{a v}^{3}+\frac{42}{9} \frac{V_{A}^{2} \cdot \gamma_{p h}}{k_{B} T(t)} t C_{M e} D_{0 M e} \exp \left(-\frac{E_{D M e}}{k_{B} T(t)}\right)\right]^{1 / 3}
$$

where $r$ is nanoparticle radius, $r_{a v}$ is is the mean value of nanoparticle radius, $\gamma_{p h}$ is surface tension at the interface with the matrix, $\mathrm{V}_{\mathrm{A}}$ is atomic volume in nanoparticles, $\mathrm{D}_{0 \mathrm{Me}}$ is nanoparticle diameter before laser treatment, and $E_{D M e}$ is nanocluster formation energy.

The value of $v_{N P}$ varies from 0 to $v_{\max }=\frac{V_{A} N_{N P \max }}{a_{o b}{ }^{2} h_{F i l m}}$, which corresponds to the saturation in the volume fraction of the nanocomposite when the nanoparticle growth is no more possible. Additionally, spectral density function $g$ ( $u$, vNP) separated the effects of the specific spatial arrangement of the metal phase and the influence of its dielectric function, expressed through the ratio $\varepsilon_{\mathrm{m}} /\left(\varepsilon_{\mathrm{m}}-\varepsilon_{\mathrm{Me}}\right)$. In accordance with Bergman theory in the Bruggemann approximation, the spectral density function was expressed as follows

$$
\begin{aligned}
& \left(1-v_{N P}\right) \frac{\varepsilon_{m}-\varepsilon_{e f f}}{\varepsilon_{m}+2 \varepsilon_{e f f}}+v_{N P} \frac{\varepsilon_{M e}-\varepsilon_{e f f}}{\varepsilon_{M e}+2 \varepsilon_{e f f}}=0 \\
& g\left(u, v_{N P}\right)=\frac{3 v_{N P}-1}{2 v_{N P}} \Phi\left(3 v_{N P}-1\right) \cdot \delta_{+}(u)+ \\
& +\frac{3}{4 \pi v_{N P} u} \sqrt{\left[u-u_{L}\left(v_{N P}\right)\right] \cdot\left[u_{R}\left(v_{N P}\right)-u\right]} \cdot \Phi\left[u-u_{L}\left(v_{N P}\right)\right] \cdot \Phi\left[u_{R}\left(v_{N P}\right)-u\right], \\
& u_{R / L}=\left(1+v_{N P} \pm 2 \sqrt{2 v_{N P}-2 v_{N P}^{2}}\right) / 3,
\end{aligned}
$$

where $\Phi(u)$ is the Heaviside function, $\delta+(u)$ is a positive delta function. It should be noted that here we are talking about the dielectric function of the composite, where the predominant part of nanoparticles has the same size $r_{N P}$;

The effective dielectric function is related to the refractive index $\mathrm{n}_{\mathrm{eff}}$ and the absorption coefficient $\mathrm{k}_{\mathrm{eff}}$ through the following expressions

$$
\begin{gathered}
n_{\text {eff }}(\omega)=\operatorname{Re} \sqrt{\varepsilon_{e f f}(\omega)}, \\
k_{\text {eff }}(\omega)=\operatorname{Im} \sqrt{\varepsilon_{e f f}(\omega)} .
\end{gathered}
$$

The distribution of nanoparticles is determined by the nanoparticles dispersion and the nanoparticles mean radius. Laserinduced changes in these parameters lead to modifications in the volume fraction of the metal phase and, therefore, modify spectral characteristics of the nanocomposite thin films. 


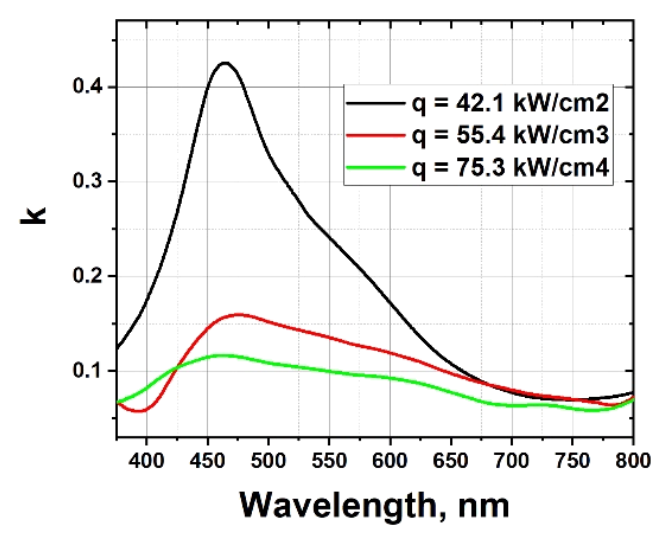

Figure 1. Calculated extinction coefficient as a function of laser wavelength.

The calculated spectral characteristics (see, for instance, Figure 1) depended on the initial size distribution of nanoparticles and were compared with experimental measurements performed for the $\mathrm{TiO}_{2}$ and $\mathrm{SiO}_{2}-\mathrm{based}$ nanocomposite films. The parameters of the nanoparticles size and distributions were controlled by the scanning speed, laser power, which, in turn were chosen based on our theoretical predictions.

\section{SUMMARY}

As a result of the performed experiments and modeling, several results have been obtained leading us to the following conclusions

- Depending on the experimental conditions, silver-nanoparticle containing nanocomposite with unique optical properties were formed.

- The optical properties of the obtained nanocomposite materials depend on the nanoparticle size and distribution and are efficiently controlled by laser processing in combination with the developed effective medium model.

\section{REFERENCES}

[1] F. M. Kahnert, Numerical methods in electromagnetic scattering theory, Journal of Quantitative Spectroscopy and Radiative Transfer, vol. 79, pp. 775-824, (2003)

[2] A. Trügler, Optical properties of metallic nanoparticles, Springer, Springer International Publishing Switzerland, (2011).

[3] K.-S. Lee and M.A. El-Sayed, Gold and silver nanoparticles in sensing and imaging: sensitivity of plasmon response to size, shape, and metal composition, The Journal of Physical Chemistry B, vol. 110, pp. 1922019225, (2006).

[4] S. Kasap and P. Capper, Springer handbook of electronic and photonic materials, 2nd ed., Springer International Publishing, (2017).

[5] J. Sancho-Parramon, S. Bosch, A. Abdolvand, A. Podlipensky, G. Seifert, H. Graener, Effective medium models for metal-dielectric composites: an analysis based on the spectral density theory, Advances in Optical Thin Films II, vol. 5963, p. 596320, International Society for Optics and Photonics, (2005).

[6] M.M. Sergeev, R.A. Zakoldaev, T.E. Itina, P.V. Varlamov, and G. K. Kostyuk, Real-time analysis of laserinduced plasmon tuning in nanoporous glass composite. Nanomaterials, 10(6), p.1131 (2020).

[7] H. Ma, S. Bakhti, A. Rudenko, F. Vocanson, D.S. Slaughter, N, Destouches, T.E. Itina, Laser-generated Ag nnoparticles in mesoporous TiO2 films: formation processes and modeling-based size prediction. The Journal of Physical Chemistry C, 123(42), 25898-25907 (2019).

[8] Y. Andreeva, N. Sharma, A. Rudenko, J. Mikhailova, M. Sergeev, V.P. Veiko, F. Vocanson, Y. Lefkir, N. Destouches, T.E.Itina, Insights into ultrashort laser-driven Au: TiO2 nanocomposite formation, The Journal of Physical Chemistry C, 124(18), 10209-10219 (2020). 